\title{
Moving towards smart transportation with machine learning and Internet of Things (IoT): a review
}

\author{
Ajay Kumar Dogra', Jagdeep Kaur ${ }^{2}$ \\ 'Department of Computer Science \& Engineering, Sardar Beant Singh State University, Gurdaspur 143521, India. \\ ${ }^{2}$ Department of Computer Science \& Engineering, Sant Baba Bhag Singh University, Jalandhar 144030, India.
}

Correspondence to: Assoc. Prof., Ajay Kumar Dogra, Department of Computer Science \& Engineering, Sardar Beant Singh State University, Gurdaspur 143521, India. E-mail: dogra2613@gmail.com

How to cite this article: Dogra AK, Kaur J. Moving towards smart transportation with machine learning and Internet of Things (IoT): a review. J Smart Environ Green Comput 2022;2:3-18. https://dx.doi.org/10.20517/jsegc.2021.09

Received: 29 May 2021 First Decision: 22 Nov 2021 Revised: 10 Dec 2021 Accepted: 27 Feb 2022 Published: 28 Feb 2022

Academic Editors: Witold Pedrycz, Elpiniki Papageorgiou Copy Editor: Xi-Jun Chen Production Editor: Xi-Jun Chen

\begin{abstract}
Internet of Things (IOT) is a new paradigm that is providing enormous services for the innovative technical advances by the researchers. There are so many loT applications that integrate the physical objects using internet and transfer the collected data over the network without human assistance. Further the collected data by the embedded devices in the objects is analyzed to take important decisions which help the society to use these applications in the different types of services. However, still there is lot of scope to deal with the various problems and apply loT in the different fields to maximize automation. Transportation is one of such area that determines the safety and quality of life of the people in the society. Due to population growth and development of technology, the demand for the vehicles in the transportation has been increasing continuously. This has attracted the researchers to contribute from different aspects by developing relevant applications and business models to make the transportation smart. The usage of IoT devices has increased the volume of the data and there is need to make this quantitative data as qualitative by using it to make the applications intelligent. So, by applying machine learning $(\mathrm{ML})$ techniques, these applications are becoming intelligent and capable of producing better results by addressing the issues in traffic management and providing safety to the residents. Many researchers have shown keen interest in such techniques and their approaches have helped to find the solution for relevant issues. This paper provides the readers an overview of the loT technology and application of the ML algorithms proposed by various authors. The focus is that the future researchers can identify the open issues and challenges and adopt various means to work beyond that. In this paper there is an introduction of development of new applications that have used the data
\end{abstract}


from loT devices thus providing meaningful outcome and make the things intelligent via ML algorithms.

Keywords: IoT, machine learning, smart, transportation

\section{INTRODUCTION}

Internet of Things (IoT) is a new model which refers to the ever-growing network of physical things that carry an Internet Protocol (IP) address to be identified and connected to internet and provides a set of new services by communicating with the other internet enabled devices and systems. IoT is a one of the latest fields due to the increase in the use of internet in the society that has attracted the interest of many researchers. It has really changed the way of life of the society as the things which were just considered as dummies have got life due to the features such as capability of being identified, the power of sensing, computing and forming a network etc. It enables large-scale of technological innovations and value-added services that will enable the users to interact with the Things that have the capability of being identified and provide the information as per the embedded devices in it. There are number of IoT applications that can be classified into different categories such as smart cities, transportation, health care system, retailing, agriculture logistics and remote monitoring. As to focus on the various research issues, there is a need to understand the meaning of IoT and to find out that how it affects the daily life as per the new business models. We are still in initial stages, where the meaning of IoT is being taken as per individual's vision and requirements ${ }^{[1,2]}$. This is the reason that there is no universal definition of IoT and we do hear of various terms that may be used for IoT like Web of Things (WoT), Machine to Machine (M2M), Cloud of Things $(\mathrm{CoT})$ etc. Though all these terms are equivalent but as per the opinions of authors these terms reflect the interpretations with respect to their vision.

The data collected through various devices in transportation is analyzed and the application of machine learning (ML) techniques on it, is heading towards the realization of smart transportation. The complex interactions among roadways, transportation traffic, environmental elements, and traffic crashes have been explored by the various researchers. In this paper, the overview of the various approaches applied using ML techniques have been presented.

\section{INTERNET OF THINGS}

The IoT is a concept that describes the idea of connecting the various physical objects to the internet thus developing the capability of being identified by the other devices and sharing the data. It becomes very close to RFID as the method of communication irrespective of the fact that it may also include other sensors and wireless technologies or QR codes. The significance of IoT is that an object can represent itself digitally and it becomes an active element of the network thus passing the useful data to the other devices in the neighborhood. This passing of information becomes possible through M2M communication. The possession of these features in the objects makes them intelligent. The capability of sensing, connecting, storing, computing etc. is acquired from various standards and technologies. Such capabilities in the objects along with other technological developments help in the integration of the things and make the IoT workable. But the diversification in deployment of technologies and standards give rise to the significant challenges in accomplishment of full connectivity thus causing complexities in integration as the major issue in IoT. The Things need to communicate with each other to extract the useful information. The type of communication that takes place in IoT can be divided in two forms:

1. Short-range like Zigbee, Bluetooth and WiFi (All Wireless);

2. Wide-range using mobile networks like WiMAX, GSM, GPRS, 3G, 4G, LTE. 
Figure 1 shows the application of IoT in the various fields. We do find IoT in all applications, which use internet as a common communication platform, regardless the objective of interest, like transportation, production, management of resources, home or business.

The main task of the IoT devices is to collect the data, M2M communication, and in some of the cases it pre-processes the data before sending it to other devices or cloud. The selection of IoT devices is done by keeping in view the power, cost and energy consumed while designing. The continuous collection and exchange of data by IoT devices results in Big Data. So, an IoT infrastructure has to implement the handling, storing and then to analyze the bulk data collected by the devices. The IoT platforms not only store and analyze the data but these are also capable of monitoring and managing the nodes. In addition to this there are some applications where the IoT devices process the data and which usually happens in the centralized node in cloud computing infrastructure. The data can move to the end networking elements for processing that gives a new model called as Edge Computing. As these devices are the basic devices, so are not able to handle the complex computational tasks. In such cases, a node is required which can upload all the data collected by IoT devices to a central cloud node thus minimizing the overhead. This node should possess adequate resources as to accomplish this task. The solution was given by authors ${ }^{[3]}$ by introducing a new term called as fog nodes. The fog nodes are having the capabilities of handling big data by providing the storage, computing and network required for such applications. Further this data stored in the various cloud nodes can be accessed for advance analysis and this can be done by using various ML techniques and sharing the same among other devices. It will help in the creation of new smart applications. Such IoT applications for a smart city have been developed which can be grouped in following categories ${ }^{[1]}$.

\section{Smart homes}

Now a days most of the homes are equipped with various appliances, such as refrigerators, washing machines, microwaves, smart TVs, LED bulbs, and other different type of electronic gadgets. These gadgets have the capabilities of transferring the data and can communicate with authorized users through internet. This can provide the users the flexibility of managing these devices irrespective of their physical presence in the home. The new applications have also been developed for providing various operations related in dayto-day activities in a smart home.

\section{Health-care assistance}

The monitoring of the patient for his health is being done with the help of new devices. For example, the sensors installed in the plasters can keep reporting the state of injury of the patient and the information collected can be examined by the doctor in the absence of the concerned patient. Wearing bands with sensors can be used to collect the various health related data from the human body and this information can be uploaded and shared with the concerned doctors to monitor the health of the patient.

\section{Smart transportation}

The most crucial application as there is urgent need to monitor the transportation due to the increase of vehicles in the urban areas. The modern vehicles are equipped with the various sensors and devices. These devices interact with the devices installed on the roadside of the city and even share the vital information through internet using mobile devices and this data can be used by the cloud nodes to share the crucial instructions to the other vehicles on the road. It is now possible to offer optimized route to the different category of vehicles, parking slots, and other useful information to public that can result in safe driving, energy conversation and avoid congestion on the roads. 


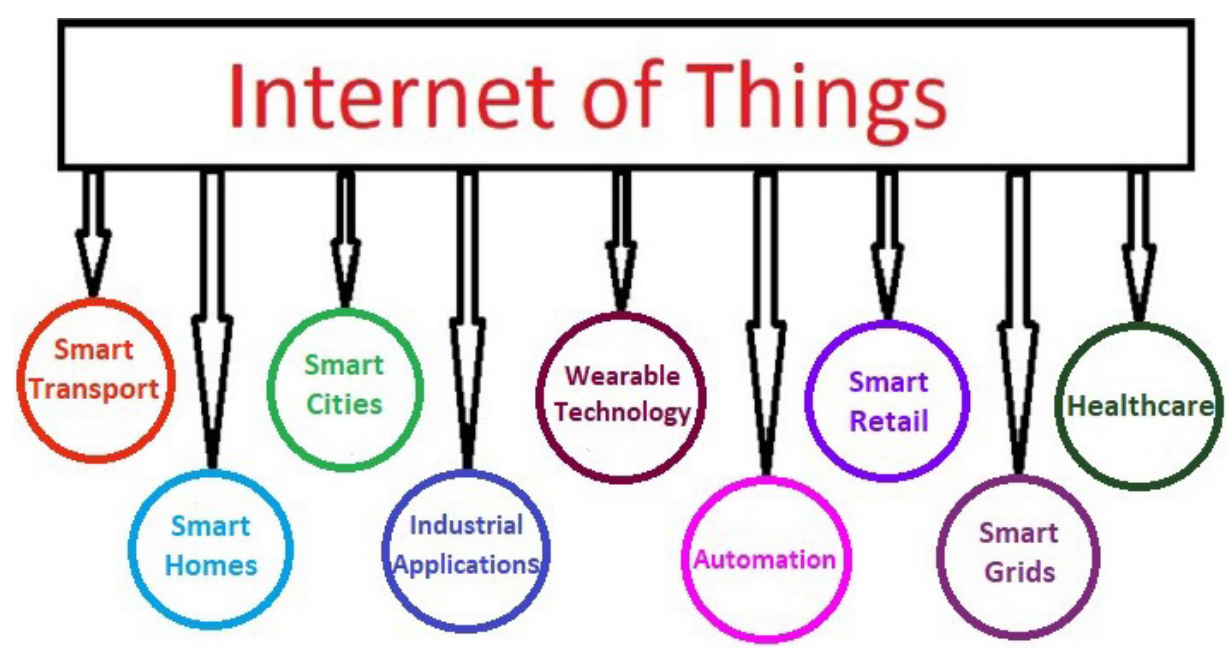

Figure 1. Use of loT in various fields. loT: Internet of Things.

\section{Environmental conditions monitoring}

Environmental conditions are monitored by the wireless sensors installed throughout the city and it makes the perfect infrastructure to collect the data required to fulfill the said objective. Many devices can be installed which can sense the pressure, humidity or wind speed to forecast the weather conditions and help the society to manage the day-to-day work schedules accordingly. Some more smart sensors can also be used to monitor the air and water pollution in the cities. The pollution data record can help the government and non-government organizations to find out the cause of any rise in the pollution levels.

\section{Logistic}

The trend of online shopping by the customers is growing day by day so there is frequent use of smart RFID tags; the status of consignments on the way from the suppliers to the customer or to the warehouses can be watched thus reducing cost and time in the overall procedure of logistics. Further the use of smart packaging can offer additional features which can take care of brand authenticity and assure the quality of the products.

\section{Security and surveillance systems}

Installation of the Smart cameras keep on monitoring the day and night life $(24 \times 7)$ on the streets and roads of the city. Keeping a close watch on the activities taking place throughout day and night keeps the miscreants away and helps the police department to control the crime in the city. In case of any incidents of theft or crime, the video obtained from the cameras and other sensors provide the vital information to identify the suspects.

\section{Challenges in loT}

IoT is being applied in the various fields and as it is in its nascent stages so the challenges are being encountered in its deployment. The number of internet users worldwide are growing day by day and the use the web regarding browsing the website, use of E-mails and other relevant information through use of social sites and other applications are growing at a fast pace. The domain of internet is growing day by day and has reached almost every section of society irrespective of the geographical location. The contribution of such users in one or the other way has increased in traffic on the network. The time is not far away when the internet will be used to access each and everything that a human will come across and IoT is playing a vital role in its automation. 
There are various challenges that are being faced due to the uploading of data by devices like sensors, actuators, and smart appliances that upload the collected data on the internet thus increasing the traffic. So, the main challenges that are being faced is concerned with traffic loads. The authors ${ }^{[4]}$ have investigated the traffic issues of M2M communications on LTE focusing on the challenges imposed on access channel and traffic channel of a radio access network. Further, the advantages and disadvantages of solutions proposed by the various researchers were presented by the authors. They were keen to find out the issues imposed by M2M communication on cellular LTE and LTE-A networks. The major problem of overload in access channel in M2M communication was investigated by the authors and the solutions were proposed. They described the various schemes along with the comparison. A possible modification wherever required was also presented. In addition to this, the second issue of overload in the data channel imposed by M2M communication was also investigated. The proposed 3GPP technologies were investigated and increase in the LTE and LTE-A was suggested by the authors.

In another effort to develop the new traffic models, protocols and enhance the capabilities of the network, the author ${ }^{[5,6]}$ have proposed the simplification of the existing IP architecture as to enable the seamless connectivity and management of heterogeneous network environment.

There are some other challenges which are being faced in the development of IoT:

- Identification and addressing of devices;

- Mobility;

- Interoperability;

- Security;

- Privacy;

- Scaling issues;

- Energy efficiency.

\section{Technologies in loT}

There is a need of technological developments in IoT but some of the technologies in which major work has been done and are used in this area are briefed below:

- RFID tag:

Its major use is in transport and logistics to identify the vehicles. It becomes easy to make use of RFID tags as the readers collect the useful information which can further be passed to the device or database as per the requirements. The use of type of technology depends upon the communication range and frequency required for the applications.

-WIFI:

It is the most common way of accessing internet and is used frequently by every section of society. Its low cost and highly portable feature attract most of the users for various indoor and outdoor applications. But still there are some special cases where it may not be used due to some privacy or security concerns.

- BAR and QR code readers:

The cost of BAR \& QR code readers is low that enables its usage in various products or goods. The implementation and use of this technology is very easy. It is mostly used for identification and scanning the price of the items in the stores. 
- Smart phones:

Keeping in view the advancement in the technology, the smart phones are affordable by almost everyone in the society. This hand-held device is not only a phone but it can be used as a tool to control the various important operations through internet. Smart phones are embedded with various types of sensors that allow the collection of important data. Its portable nature allows the users to be connected with the world irrespective of their physical location.

- IoT Infrastructure:

IoT has been used in different applications and the components of the infrastructure ${ }^{[1]}$ used in most of the applications are as below:

1. IoT nodes: These nodes are the IoT devices such as sensors and actuators that collect the data and exchange it with the other nodes. The data is even uploaded on the fog nodes for further computations. The IoT nodes may possess the capability of preprocessing the data and computing some basic tasks.

2. Fog nodes: These nodes are geographically distributed and help to handle the big data collected by the IoT nodes. It further offers computing, storage and advanced networking capabilities to the IoT devices.

3. Cloud nodes: The collected data by IoT nodes is stored in the datacenters. This information is shared to the other devices and applications. The advanced computational tasks can be handled at this level. The back-end software of the applications can be executed by using ML.

\section{Smart city applications}

Smart healthcare: The health of the individual is the top most priority now a days. There are so many options available for the patients if the procedure for early diagnosis becomes easy. Smart healthcare plays an important role for early diagnosis of the disease. The smart wearable devices and sensors helps to monitor the health of the patients and the dynamic data regarding the current status of the patient can be sent to the doctor or the hospitals with the help of communication technologies such as Zigbee and Bluetooth. Lack of precision is the challenge which is being taken as the area for future work and provide the accurate information which can be used to critically monitor the health of the patients.

Smart grid: Power supply is the major requirement for society and its management is a herculean task for the power management authorities. The power supply is managed through installation of smart meters and readers. The use of WiFi, Z-wave, Zigbee etc. has made it easy to efficiently manage the power supply to the society. The estimate of the future requirements helps to cater the growing power supply needs of the residents.

Smart transportation: Smart transportation has changed the way of managing the traffic in the cities. It enables the dynamic management of traffic and provides the efficient and safe routes to the traffic. The use of IoT devices such as sensors, RFID cards, smart vehicles, cameras and other wearable electronic smart gadgets by the passengers has enabled the dynamic management of the increasing traffic in the city. The latest communication technologies like RFID, 3G, $4 \mathrm{G}$ and $5 \mathrm{G}$ has made it easy to transfer the data between the IoT devices and the servers as and when required. It has become easy to manage the traffic automatically but at the same time the failure of the technology or delay/loss of data may cause serious issues. So, researchers are adopting the various methodologies and developing the models as to provide the reliable solution.

Cities today use a range of technologies to manage traffic signals, video cameras, RFID readers and more to control the traffic in the city. With the growth of ITS, the number and types these connected components is increasing. The arrival of connected vehicle technology and the future of autonomous vehicles is resulting 
in an increasing demand for maximum network bandwidth and communication technology.

Authors ${ }^{[7]}$ developed an edge based multi-agent auto communication algorithm using IoT for controlling the traffic lights for smart cities. As per authors the algorithm is an innovative adaptive global traffic light control method that combines multiagent auto communication protocol with MARL (multi agent reinforcement learning) to allow an agent to communicate the learned strategies with others for achieving global optimization in traffic signal control. Further, considering the limitations of the capabilities of network transmission bandwidth, a predictable edge computing architecture for industrial deployment on IoT was also presented in the paper. The various methods of traffic control signals have been summarized in Table 1.

Smart governance: To manage the citizens and run the system efficiently, there is urgent need to make smart policies and implement the same in the city. The smart devices help the management to keep close watch on the people and the establishments and act accordingly as to control the law and order in the city. The collection of data through the smart devices helps them to keep close vigil on the citizens. In case of any emergencies or law and order issues, the information collected through these devices help the administration to smartly narrow down the search and catch hold of the culprits.

\section{MACHINE LEARNING}

The concept of ML is basically derived from Artificial Intelligence (AI). The implementation of the concepts of AI becomes feasible through ML. The tasks like predictions, classification, pattern recognition, clustering, etc. are performed by computer systems through ML. The sample data collected through various means carries certain measurable characteristics which are known as features and the task of the ML algorithm is to find a correlation between these features and output values generated which are basically termed as labels ${ }^{[8]}$. After this the information obtained during the training phase is used for making the decisions or identify patterns based on new data. There are lot of problems related with regression, classification, clustering and association where the role of ML comes into play. The classification of ML algorithms based on the learning style is divided into four categories ${ }^{[1]}$.

Supervised learning: Supervised learning is the method where the algorithm learns from the labeled training data say if there are input variables say $(\mathrm{X})$ and the output variable $(\mathrm{Y})$ and then there is an algorithm that learns the mapping function from input to the output generated $[\mathrm{Y}=\mathrm{f}(\mathrm{X})]$. The task is to approximate the mapping function so that whenever the new set of input values are given, the output $\mathrm{Y}$ will be predicted for the input data. The term supervised learning comes from the fact that the algorithm learns from the training dataset. The problems like weather forecasting, life experiences case studies, and some predictions like population growth are dealt with supervised learning techniques. The algorithms used for processing such computations are like Linear Regression or Random Forest. There are many other issues such as classification problems like speech or digit recognition, credit card fraud detection which can be addressed by using supervised learning algorithms. The supervised learning works in two phases, the first one is training phase and the other is testing phase. The data sets need to have known labels which are used for the training phase. The algorithms read the data and learn by identifying the relationship between input data and labels for predicting output values of testing data ${ }^{[0]}$.

Unsupervised learning: Unsupervised learning is a type of ML that will learn from test data that has not been labeled and classified. So rather than responding to feedback, this technique relies on the structure of data that identifies the common elements in the data and responds on the basis of existence or absence of common elements in each new frame of data. As, in this type of learning no labels are available so 
Table 1. Traffic signal control summary ${ }^{[7]}$

\begin{tabular}{|c|c|c|}
\hline Method & Advantages & Disadvantages \\
\hline Fixed time & $\begin{array}{l}\text { Traditional way, easy deployment and } \\
\text { implementation }\end{array}$ & Static method and non-adaptive to intersection changes \\
\hline $\begin{array}{l}\text { One traffic light } \\
\text { optimization }\end{array}$ & $\begin{array}{l}\text { As per the current scenario, the signals can be } \\
\text { adjusted dynamically }\end{array}$ & $\begin{array}{l}\text { AS the traffic in cities consists of multiple traffic light signals, so } \\
\text { can't provide global optimization }\end{array}$ \\
\hline $\begin{array}{l}\text { Optimization of multiple } \\
\text { traffic lights }\end{array}$ & $\begin{array}{l}\text { Optimizes the traffic signals globally at multiple } \\
\text { intersections }\end{array}$ & $\begin{array}{l}\text { Difficulty in implementation and deployment. Algorithms can } \\
\text { further be optimized }\end{array}$ \\
\hline
\end{tabular}

algorithms work itself to discover the presence of some related structures in the input data. Unsupervised learning algorithms of this category works for identifying patterns on testing data and try to form the clusters that will further tend to make the prediction for future values ${ }^{[10]}$.

Semi-supervised learning: The above mentioned two techniques are combined to form this category. In this type of learning there is large amount of input data but some of the data is only labeled. So the combination of labeled and unlabeled data is used in this type of learning. The working of this learning is similar to unsupervised learning but the labeled data adds the improvements that creates the difference in $\mathrm{two}^{[8]}$.

Reinforcement learning: Reinforcement learning is a technique where an agent learns in an interactive environment by hit and trial method using feedback from its own actions and experiences. By doing this these machines and software agents automatically maximize its performance by determining the ideal behavior within a specific context. The specific type of problem defines the reinforcement learning and the solutions corresponding to this are classified as reinforcement learning algorithms. Based on the current state of problem environment, the agent selects the best action to be taken to maximize the total cumulative reward. The repetition of this step in the problem is known as a Markov Decision Process. The authors ${ }^{[0]}$ have used reinforcement learning for various applications that include skill acquisition, AI gaming, robot navigation, and other real-time decisions. There are two major parameters of concern that has to be taken care of before using the ML technique:

- How computationally intense the technique is;

- And, how fast it will generate the result.

So, choosing the algorithm depends upon the above two parameters. While applying it to the real time applications, it must be taken care of that selected algorithm should be efficient to observe the changes that takes place in the data input so that the desired output be obtained timely.

\section{Machine learning for smart transportation}

The installation of IoT devices and their connectivity enables these devices to the exchange the information. This results in collection of data and it keeps on growing day by day. Some of the applications are such that the IoT devices trigger some action depending upon the programming and the feedback received through data collection. In order to create smart applications, the role of human is important as the collected data has to be analyzed for getting meaningful information out of it. What is expected of IoT devices is that these devices should not only collect and communicate with the other devices but should learn from the collected data and take the context-based decisions accordingly. This act of devices created the term Cognitive IoT. There is a need for IoT devices which are intelligent enough to create automated smart applications with automated allocation of resources, communication and network operations. In order to improve the IoT applications significantly, the use of ML algorithms turns out to be quite helpful as it optimizes the allocation of resources, resolving congestion and network optimization. 
The increase of devices with time increases the collected data manifolds and to handle the Big Data which becomes difficult for conventional databases, a special infrastructure is required which will deal with the structured and unstructured data and analyze the same. There are many ML algorithms which can be applied to deal with the Big Data efficiently. Some of these algorithms used in smart transportation applications have been classified ${ }^{[1]}$ as per the learning types shown in Figure 2:

1. Supervised learning algorithms:

- AdaBoost;

- Bayesian network seasonal autoregressive integrated moving average;

- Decision tree;

- Feed forward neural networks;

-k-nearest neighbor;

- Logistic regression;

- Random forest (RF);

- Regression tree;

- Super vector machine.

2. Unsupervised algorithms:

- Fuzzy C-means;

- K-means;

- Markov random field.

3. Reinforcement algorithms:

- Convolutional neural networks (CNN) \& deep CNN;

- Couple hidden Markov model;

- Deep brief networks;

- Fully connected networks

- Markov decision process;

- Regression tree.

The rate at which the vehicles are increasing on the roads is even more than the rate of overall population. It is leading to congested roads and it has become dangerous to use these roadways for the travel. It becomes difficult to address this issue by increasing the number of roads as it becomes very costly to construct the roads and then thin of catching the regular increase of vehicles. The option is to manage the traffic by analyzing the traffic data available on the highways. The traditional data analytics solutions may not work as the scale of data generated in the transportation system is very high. So, ML is a data-driven solution that can take up the challenge as per the needs of the system. ML methodologies learn by referring the historical data and extracting the patterns out of it to establish a relation depending upon the system behavior and then acts to automate the system to generate output.

There are many IoT applications used in smart transportation and ML algorithms are applied to deal with the type of challenges faced in smart transportation. Following are the major categories of smart transportation challenges which are dealt by the authors by applying ML and non-ML based techniques.

- Route optimization; 


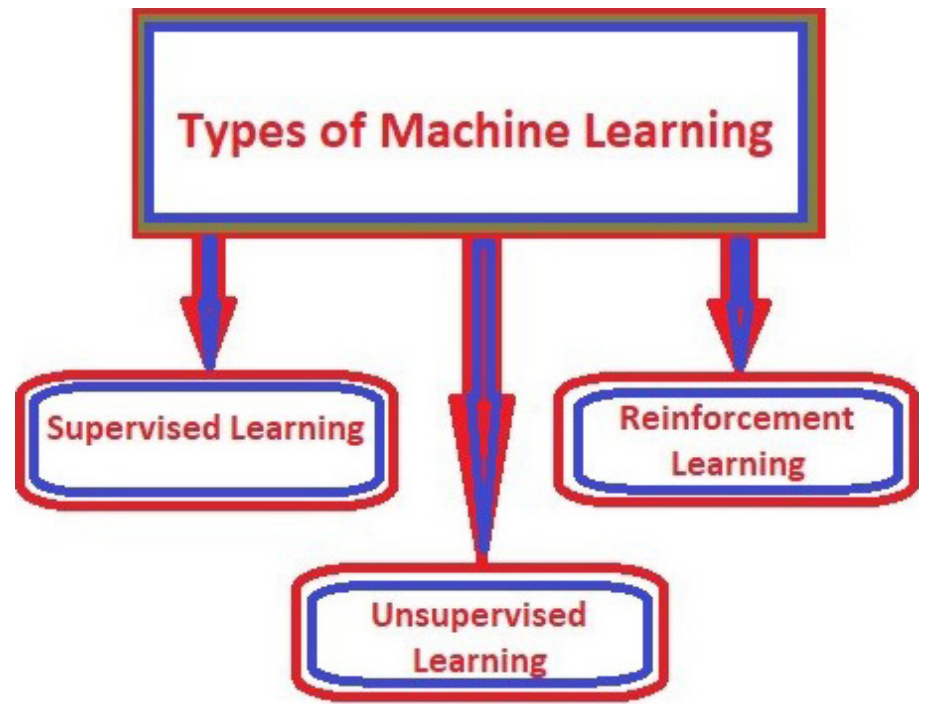

Figure 2. Types of machine learning algorithms.

- Parking issues;

- Lighting;

- Detection of accidents;

- Evaluating road conditions;

- Infrastructure.

\section{Route optimization}

Irrespective of the city being urban or rural, the number of vehicles has been increasing day by day thus causing a common issue of traffic congestion. To avoid the traffic jams and resolve the congestion issues route optimization method is applied to find the optimal route for the desired destination. It will lead to minimize the congestion in the traffic along with saving energy and travel time. It will further reduce the vehicle emissions thus contributing to lower the environmental pollution. Many authors have been working on route optimization and have proposed solutions by using various ML approaches. To predict the travel mode of individuals in the city Luxembourg, the authors ${ }^{[9]}$ presented four ML methods namely net-MLP, multinomial logistic regression, AI neural net-RBF and support vector machines. These methods used the traveler's characteristics, mode of travelling and the information related to their residence and place of work. The results obtained showed that the artificial neural networks perform better than the other alternative approaches. Authors ${ }^{[1]}$ have used MDP algorithm and V2V communication for group routing so that the traffic congestion can be minimized. The authors proposed the solution by forming the group on the basis of vehicle types and the behavior of the drivers. The developed algorithm approach assigned the routes to the vehicles to avoid the congestion. The algorithms $\mathrm{k}$-means and $\mathrm{DBN}^{[12]}$ have been combined to optimize the traffic network configuration. Authors ${ }^{[13]}$ have challenged short term and long-term traffic predictions by comparing the performance of four ML methods i.e., RF, a baseline predictor, FF-NN, and a regression tree. Traffic congestion predictions were dealt by the authors ${ }^{[14]}$ by comparing a logistic regression with SVM, a decision tree, an RF, and a MLP method. The author proposed that the logistic 
regression gave an early prediction of the congestion in a simpler way as compared to the other three algorithms.

A vehicular ad-hoc network based algorithm ${ }^{[15]}$ is implemented for enhanced route planning. A mobile application was developed by the authors that collects traffic data and then proposes the optimal route to the commuters that will save the travelling time or save the fuel.

\section{Parking issues}

The development of parking applications is done to keep the track of available parking space and offer the same to commuters to park their vehicles. To find out the availability of parking space the use of IoT devices have helped to find out parking slots that have already been occupied and then send this information to the centralized system. The authors ${ }^{[16]}$ presented a lifecycle general approach using Lifecycle Modeling Language for IoT enabled smart city service systems. It has been demonstrated by the authors in the smart parking for a shopping mall in the city. As presented in their work, the proposed scenario allots the parking slot to the drivers as soon as they drive towards the any of the entrances available in the shopping mall.

ML algorithms can also be applied to detect the free parking slots and the authors ${ }^{[17]}$ have used CNNs on the data that is taken from the installed cameras in the parking area to find out such free slots. These images from the cameras are processed ${ }^{[18]}$ where the authors have used the combination of SVM and MRF algorithm to find out such free slots. Author ${ }^{[19]}$ have used ultrasound sensors to detect the parking slot availability and this information collected by Wi-Fi module will be send to a cloud server. The users can check the availability of the parking space through the mobile application.

A combination of the software and hardware is proposed by authors ${ }^{[20]}$ to find the free slots in the parking and convey the same to the users. The process of detection of cars in the parking slot is done by the magnetic sensors and this information is send to the remote servers by the gateway devices located on the road side. The nearest parking slot is offered to the driver through the algorithm developed by the authors in the proposed methodology. The genetic algorithms are executed to find out the nearest parking slot for the vehicle looking for it. There are some authors who have also used non-ML techniques to find the solution to the parking related issues.

The authors ${ }^{[21]}$ have proposed the models that predicts and forecasts the information related to parking by studying the data generated by the various parking systems of the city Riyadh. As proposed the information may be useful for improving the various parking areas of the city. The authors have introduced the recurrent neural network method and have further improved the quality of the model by including exogenous variables concerned to the calendar effects and weather conditions.

\section{Lighting}

One of the major parts of smart transportation services is smart street lights (SSL). As the well-planned residential areas are being established in the modern cities so the installation of the lights in the roads and streets has increased. Thus, the management of lights helps in reducing the consumption of energy and contributes a lot in establishing a smart city. The authors ${ }^{[22]}$ have implemented the SSL based on IoT devices. To make the street lights smart, the addition of light sensors, IR sensors, GPS and wireless communication module is required and this will enable the lights to provide the vital information as per the requirements of the concerned areas. The location of lights can well be known by the GPS system and the maintenance of the installed equipment can easily be taken care of. A simple approach is given by authors ${ }^{[23]}$ where they have installed IR sensors and IR LeDs in the lights which will sense the sun rise and sun set as to trigger the 
switching as required. The lamps with such sensors can also detect the passing cars and pedestrians to control the switching of lamps dynamically.

Authors $^{[24]}$ have proposed a system where each lamp post is taken as a Wi-Fi hot spot. It will transfer collected information to central web server and the switching of lights will be dynamic depending upon the surroundings. Moreover, the installation of cameras on the poles will enhance the security of people by keeping record of surroundings and avoid any foul play by the miscreants.

\section{Detection of accidents}

Accident detection and prevention is the most important activity for any type of city as the human life is most important and is required to be given the top most priority. To avoid road accidents, the best option is to make the driver to stay alert throughout the driving period. If the driver is timely alerted about the forthcoming critical situations the he can act timely to avoid the accidents. If the accidents can be avoided then it will ultimately save the lives and reduce the traffic congestion scenarios. The use of ML has resulted in detection of road accidents and further identified the situations that could lead to new accidents. The timely notification to the drivers will help them to act accordingly and avoid the accidents. Authors ${ }^{[25]}$ have developed a method using CHMM in which the drivers of the vehicle share vital information as to avoid the accidents. Authors ${ }^{[26]}$ have performed the real time detection of the accidents by obtaining the data from the road side sensors. The algorithms like FF-NN, a regression tree, and k-NN are used are used to analyze the data and get the above results. A camera based blind spot detection system was developed based on fully connected networks ${ }^{[27]}$ to detect the objects in the blind spot and providing warning to the drivers of smart vehicles. The authors ${ }^{[28]}$ have worked to identify the accident-prone areas so that the warning to the drivers approaching such area can be given to avoid accidents. The authors ${ }^{[29]}$ have proposed an IoT cloud platform that will visualize the traffic and provide prior information to the driver for the sudden slowdown of traffic.

\section{Evaluating road conditions}

It has been observed that the road anomalies lead to the major accidents as drivers may not be aware of sudden pot-holes, bumps or bad patches in the road. If the drivers are informed about the occurrence of any such bad patches, it will definitely avoid the accidents. The challenge of detection of road anomalies can be dealt with the ML techniques and some researchers have contributed by using these techniques.

The authors ${ }^{[30]}$ have used the accelerometer data obtained from the mobiles and processed using FF-NN to detect the pot-holes in the roads. A same type of approach is used by another author to detect the road anomalies by using the algorithm called as k-means. Deep learning techniques applied for pavement distress detection have been reviewed in details by author ${ }^{[31]}$. The reviewed studies based on CNN and DCNN compared on some parameters show that in most of studies with reference to pavement image classification, the CNNs show the best result.

\section{Infrastructure}

The capabilities of the system can be expanded by changing the infrastructure associated with the Intelligent Transportation System. As the technology in IoT has improved, so the modern transportation system has benefitted a lot out of it. The authors in Ref. ${ }^{[32]}$ have presented the advantages and disadvantages of newly released Bluetooth standard 5.0 by Bluetooth Special Interest Group which is being considered as a major development in short range wireless communication technology. As per the comparison done by the authors in the said article, the Bluetooth 5.0 standard uses frequency hopping technique to cover the range up to $200 \mathrm{~m}$ at $2 \mathrm{Mb} / \mathrm{s}$ with a latency of less than $3 \mathrm{~ms}$. It can hold up-to 255 bytes of message size. The new standard allows data exchange among the various connected appliances and smart devices used in the IoT. 
Its greater speed, extended broadcast capacity and longer battery life due to use of fewer energy resources are the prominent advantages making it suitable to be used in IoT devices.

The authors in Ref. ${ }^{[33]}$ have focused use of electric vehicles (EVs) that have played a significant role in the development of smart cities. Among the EVs, the electric bicycles (e-bike) that uses electric motor powered by the rechargeable batteries have been considered by the authors for providing a fuzzy based solution for optimizing and managing energy consumption in these e-bikes. The results produced by the authors show that that the proposed approach outperforms the other methods and significantly prolongs battery life. Such kind of development in the infrastructure will highly contribute in achieving the goal of development of smart cities.

The authors ${ }^{[34]}$ have developed a Bike Sharing System which provide the services in order to make a prediction with a high probability that the bike may be returned at the station where the user is waiting to acquire one. The goal was to analyze the feasibility of these services and to put forward the idea of using ML methodologies. The authors applied the different ML models on the data logs of the three identified features i.e., Station Profile All, Station Profile and User Profile to make the said predictions.

The authors ${ }^{[35]}$ have proposed a new way of communication by simulating vehicle to vehicle communication based on IoT. In this framework, the cars will use the GPS to provide the information of their position and then exchange the vital data like speed, location, movements etc. with the neighboring cars. The data uploaded on the server will aware the drivers of the approaching cars to act accordingly in case of sudden speed change or traffic congestion. The author ${ }^{[36]}$ have combined the hardware consisting of RFID tags and software to uniquely identify the vehicles. The installation of IR sensors can help to keep the record of passengers entering and leaving the bus. The collected information can be uploaded on the cloud and then the same can be displayed on the bus stops to make the passengers aware of the status of the buses.

\section{ISSUES \& CHALLENGES}

The complexity in the IoT based systems is obvious due to the deployment of various technologies to exchange the information among embedded devices. Use of IoT devices in various aspects like health, environment, transportation, agriculture, security etc. has impacted the human life. Thus, consideration of all the issues and challenges related to IoT and its implementation becomes very important. In transportation, the authors have highlighted the issues arising due to increase in number of devices and use of different technologies by these devices do add the complexity in integration of technologies. Large amount of data storage issues along with the heterogeneous environment create the issues which need to be looked into by the researchers. The privacy and the security issues has always been the bone of contention for implementing IoT based models. The researchers have proposed the different models to address these issues but still a lot of work is needed to be done in order to provide a secured model that can deal with most of the challenges in use of IoT in various applications in day-to-day life.

Standardization of communication techniques and the various IoT architecture is the need of the hour. Most of the issues pertains due to the diversification of architecture and communication among the IoT devices. There are few standard bodies which are looking into the said issues and working towards the development of such standards.

\section{FUTURE SCOPE}

The review of various papers in the area of smart transportation has given the scope for future work. As per the proposed model by authors in blind spot detection, the system still needs to be improved as far as the 
characteristics of connectivity and the detection of blind spot is concerned. The application of deep learning algorithms needs to be considered for detection of blind spot. In the history of research studies, it shows that the wireless sensors networks have been used extensively in the traffic management systems. The monitoring of the traffic can be done by the deployment of various types of sensors. The selection of sensors in respect of its power consumption, sensitivity, reliability, cost etc. becomes critical. The authors ${ }^{[37]}$ have proposed system that uses WiFi to communicate between IoT devices, but their energy consumption and solution to recharge these devices have not been taken care of. So, an alternate solution such as use of solar power or use of some additional available means need to be considered. Moreover, the proposed model by the authors has been tested only in the context of the single-lane road. However, as most of the network of roads has been converted into multiple lanes so, it would be beneficial to test the system in a multi-lane scenario to identify the false detections. The communication between the various traffic signals in the city can be considered to provide the latest information to the commuters and control the traffic system.

\section{CONCLUSIONS}

A literature review on use of Internet of Things with respect to transportation and applying ML techniques on it was explored and discussed. In this paper, a number of approaches by the various authors using different type of $\mathrm{ML}$ algorithms were stated. As per the different proposed algorithms, the researchers have contributed a lot to make the transportation smart by using the data collected from the IoT devices and applying ML algorithms on it. We believe that the published work by various authors contribute in making the transportation smart and handle the issues arising out of increase in traffic on the roads. It is seen that still there is scope for ML coverage in the different areas in transportation, specifically in traffic lights at the various intersection points in the city. The parking applications can be further explored and the use of appropriate ML techniques may turn out to be a boon for the increasing number of vehicles in the city. Moreover a few features have been used to identify the congestion, road anomalies and accident prevention models. Although lot of progress has been made in the field of transportation with the use of IoT and ML algorithms but still in future some more features can be considered in order to develop the predictive models by using various IoT devices and ML algorithms. Further various models can be developed with this combination that can address the issues, arising due to increase in data in transportation system. Researchers can look for a better solution to the challenges being faced by the society where the rate of increase of vehicles on the roads is more than the rate of increase in population.

\section{DECLARATIONS}

\section{Authors' contributions}

Made contribution towards the design, study and interpretation: Kumar DA

Provided technical guidance and material support: Jagdeep K

\section{Availability of data and materials}

Not applicable.

\section{Financial support and sponsorship}

None.

\section{Conflicts of interest}

Both authors declared that there are no conflicts of interest.

\section{Ethical approval and consent to participate}

Not applicable. 


\section{Consent for publication}

Not applicable.

\section{Copyright}

(c) The Author(s) 2022.

\section{REFERENCES}

1. Zantalis F, Koulouras G, Karabetsos S, Kandris D. A review of machine learning and IoT in smart transportation. Future Internet 2019;11:94. DOI

2. Singh D, Tripathi G, Jara AJ. A survey of Internet-of-Things: future vision, architecture, challenges and services. 2014 IEEE World Forum on Internet of Things (WF-IoT); 2014 Mar 6-8; Seoul, Korea (South). IEEE; 2014. p. 287-92. DOI

3. Luan TH, Gao L, Li Z, Xiang Y, Sun L. Fog Computing: Focusing on Mobile Users at the Edge. Available from: https://arxiv.org/abs/1502.01815 [Last accessed on 28 Feb 2022].

4. Soltanmohammadi E, Ghavami K, Naraghi-pour M. A survey of traffic issues in machine-to-machine communications over LTE. IEEE Internet Things J 2016;3:865-84. DOI

5. Soret B, Pedersen KI, Jørgensen NTK, Fernández-lópez V. Interference coordination for dense wireless networks. IEEE Commun Mag 2015;53:102-9. DOI

6. Andrews JG. Seven ways that HetNets are a cellular paradigm shift. IEEE Commun Mag 2013;51:136-44. DOI

7. Wu Q, Wu J, Shen J, Yong B, Zhou Q. An edge based multi-agent auto communication method for traffic light control. Sensors (Basel) 2020;20:4291. DOI PubMed PMC

8. Mohammed M, Khan MB, Bashier EBM. Machine learning: algorithms and applications. 1st ed. Boca Raton: CRC Press; 2016. DOI

9. Kubat M. An introduction to machine learning. 3rd ed. Cham: Springer; 2017. DOI

10. Omrani H. Predicting travel mode of individuals by machine learning. Transportation Research Procedia 2015;10:840-9. DOI

11. Sang KS, Zhou B, Yang P, Yang Z. Study of group route optimization for IoT enabled urban transportation network. 2017 IEEE International Conference on Internet of Things (iThings) and IEEE Green Computing and Communications (GreenCom) and IEEE Cyber, Physical and Social Computing (CPSCom) and IEEE Smart Data (SmartData); 2017 Jun 21-23; Exeter, UK. IEEE; 2017. p. 888-93. DOI

12. Yang J, Han Y, Wang Y, Jiang B, Lv Z, Song H. Optimization of real-time traffic network assignment based on IoT data using DBN and clustering model in smart city. Future Generation Computer Systems 2020;108:976-86. DOI

13. Hou Y, Edara P, Sun C. Traffic flow forecasting for urban work zones. IEEE Trans Intell Transport Syst 2015;16:1761-70. DOI

14. S.; Neetha, T. Machine learning based traffic congestion prediction in a IoT based Smart City. Int Res J Eng Technol 2017;4:3442-5.

15. Chang I, Tai H, Yeh F, Hsieh D, Chang S. A VANET-based A* route planning algorithm for travelling time- and energy-efficient GPS navigation app. Int J Distrib Sens Netw 2013;9:794521. DOI

16. Hefnawy A, Bouras A, Cherifi C. IoT for smart city services: lifecycle approach. Proceedings of the International Conference on Internet of things and Cloud Computing; Cambridge, UK. New York: Association for Computing Machinery; 2016. DOI

17. Amato G, Carrara F, Falchi F, Gennaro C, Meghini C, Vairo C. Deep learning for decentralized parking lot occupancy detection. Expert Syst Appl 2017;72:327-34. DOI

18. Wu Q, Huang C, Wang SY, Chiu WC, Chen T. Robust parking space detection considering inter-space correlation. 2007 IEEE International Conference on Multimedia and Expo; 2007 Jul 2-5; Beijing, China. IEEE; 2007. p. 659-662. DOI

19. Gupta A, Kulkarni S, Jathar V, Sharma V, Jain N. Smart car parking management system using IoT. American Journal of Science, Engineering and Technology 2017;2:112-9.

20. Aydin I, Karakose M, Karakose E. A navigation and reservation based smart parking platform using genetic optimization for smart cities. 2017 5th International Istanbul Smart Grid and Cities Congress and Fair (ICSG); 2017 Apr 19-21; Istanbul, Turkey. IEEE; 2017. p. 120-4. DOI

21. Arjona J, Linares, MP, Casanovas J. A deep learning approach to real-time parking availability prediction for smart cities. Proceedings of the Second International Conference on Data Science, E-Learning and Information Systems; Dubai, United Arab Emirates. New York: Association for Computing Machinery; 2019. DOI

22. Jia G, Han G, Li A, Du J. SSL: smart street lamp based on fog computing for smarter cities. IEEE Trans Ind Inf 2018;14:4995-5004. DOI

23. Kokilavani M, Malathi A. Smart street lighting system using IoT. Int J Adv Res Appl Sci Technol 2017;3:8-11. DOI

24. Tripathy AK, Mishra AK, Das TK. Smart lighting: intelligent and weather adaptive lighting in street lights using IOT. 2017 International Conference on Intelligent Computing, Instrumentation and Control Technologies (ICICICT); 2017 Jul 6-7; Kerala, India. IEEE; 2017. p. 1236-9. DOI

25. Liu W, Kim S, Marczuk K, Ang MH. Vehicle motion intention reasoning using cooperative perception on urban road. 17th International IEEE Conference on Intelligent Transportation Systems (ITSC); 2014 Oct 8-11; Qingdao, China. IEEE; 2014. p. 424-30. DOI

26. Ozbayoglu M, Kucukayan G, Dogdu E. A real-time autonomous highway accident detection model based on big data processing and 
computational intelligence. 2016 IEEE International Conference on Big Data (Big Data); 2016 Dec 5-8; Washington, DC, USA. 2016. p. 1807-13. DOI

27. Kwon D, Park S, Baek S, Malaiya RK, Yoon G, Ryu J. A study on development of the blind spot detection system for the IoT-based smart connected car. 2018 IEEE International Conference on Consumer Electronics (ICCE); 2018 Jan 12-14; Las Vegas, NV, USA. IEEE; 2018. p. 1-4. DOI

28. Ryder B, Wortmann Felix. Autonomously detecting and classifying traffic accident hotspots. Proceedings of the 2017 ACM International Joint Conference on Pervasive and Ubiquitous Computing and Proceedings of the 2017 ACM International Symposium on Wearable Computers; Maui, Hawaii. New York: Association for Computing Machinery; 2017. p. 365-70. DOI

29. Celesti A, Galletta A, Carnevale L, Fazio M, Lay-ekuakille A, Villari M. An IoT cloud system for traffic monitoring and vehicular accidents prevention based on mobile sensor data processing. IEEE Sensors $J$ 2018;18:4795-802. DOI

30. Kulkarni A, Mhalgi N, Gurnani S, Giri N. Pothole detection system using machine learning on Android. Int J Emerg Technol Adv Eng 2014;4:360-4. DOI

31. Gopalakrishnan K. Deep learning in data-driven pavement image analysis and automated distress detection: a review. Data $2018 ; 3: 28$. DOI

32. Collotta M, Pau G, Talty T, Tonguz OK. Bluetooth 5: a concrete step forward toward the IoT. IEEE Commun Mag 2018;56:125-31. DOI

33. Giliberto M, Arena F, Pau G. A fuzzy-based solution for optimized management of energy consumption in e-bikes. $J$ Wirel Mob Netw Ubiquitous Comput Dependable Appl 2019;10:45-64. DOI

34. Bacciu D, Carta A, Gnesi S, Semini L. An experience in using machine learning for short-term predictions in smart transportation systems. Journal of Logical and Algebraic Methods in Programming 2017;87:52-66. DOI

35. Chowdhury DN, Agarwal N, Laha AB, Mukherjee A. A vehicle-to-vehicle communication system using IoT approach. 2018 Second International Conference on Electronics, Communication and Aerospace Technology (ICECA); 2018 Mar 29-31; Coimbatore, India. IEEE; 2018. p. 915-9. DOI

36. Geetha S, Cicilia D. IoT enabled intelligent bus transportation system. 2017 2nd International Conference on Communication and Electronics Systems (ICCES); 2017 Oct 19-20; Coimbatore, India. IEEE; 2017. p. 7-11. DOI

37. Sarrab M, Pulparambil S, Awadalla M. Development of an IoT based real-time traffic monitoring system for city governance. Global Transitions 2020;2:230-45. DOI 